\title{
Automated NDT of floating production storage oil tanks with a swimming and climbing robot
}

\author{
T.P. Sattar, H.E. Leon Rodriguez, J. Shang and B. Bridge \\ Department of Electrical, Computer and Communications Engineering, \\ London South Bank University, 103 Borough Road, London SE1 0AA,UK \\ sattartp@Lsbu.ac.uk
}

\begin{abstract}
This paper describes the design and development of a prototype swimming and wall-climbing robot that gains access to internal tank wall and floor surfaces on Floating Production Storage Oil (FPSO) tanks for the purposes of carrying out Non-Destructive Testing (NDT) of welds while the tank is in-service and full of oil. A brief description is given of the inspection environment and the three NDT techniques (ultrasonic phased arrays, eddy current arrays, and Alternating Current Field Measurement ACFM arrays) that will be used to detect weld cracks and floor corrosion and pitting.
\end{abstract}

Keywords: FPSO NDT, Swimming \& Climbing Robot

\section{Introduction}

FPSO tanks [1] are found on ships moored near off-shore oil platforms. They are used to store oil before transportation to the mainland. For structural safety and environmental reasons, it is necessary to test the welds periodically.

The main inspection task is to test the integrity of welds on plates used to strengthen the walls and floor of the tank. Currently they are inspected manually by emptying the tank of product after thoroughly cleaning it. Human operators then enter the tank to perform ultrasonic NDT. There is a large cost associated with the cleaning and inspection tasks. A pair of tanks are emptied, cleaned and inspected in 3-4 weeks with 60-70 man-days work and costs between $£ 25-30 \mathrm{k}$. A pair of FPSO tanks and ballast tanks 


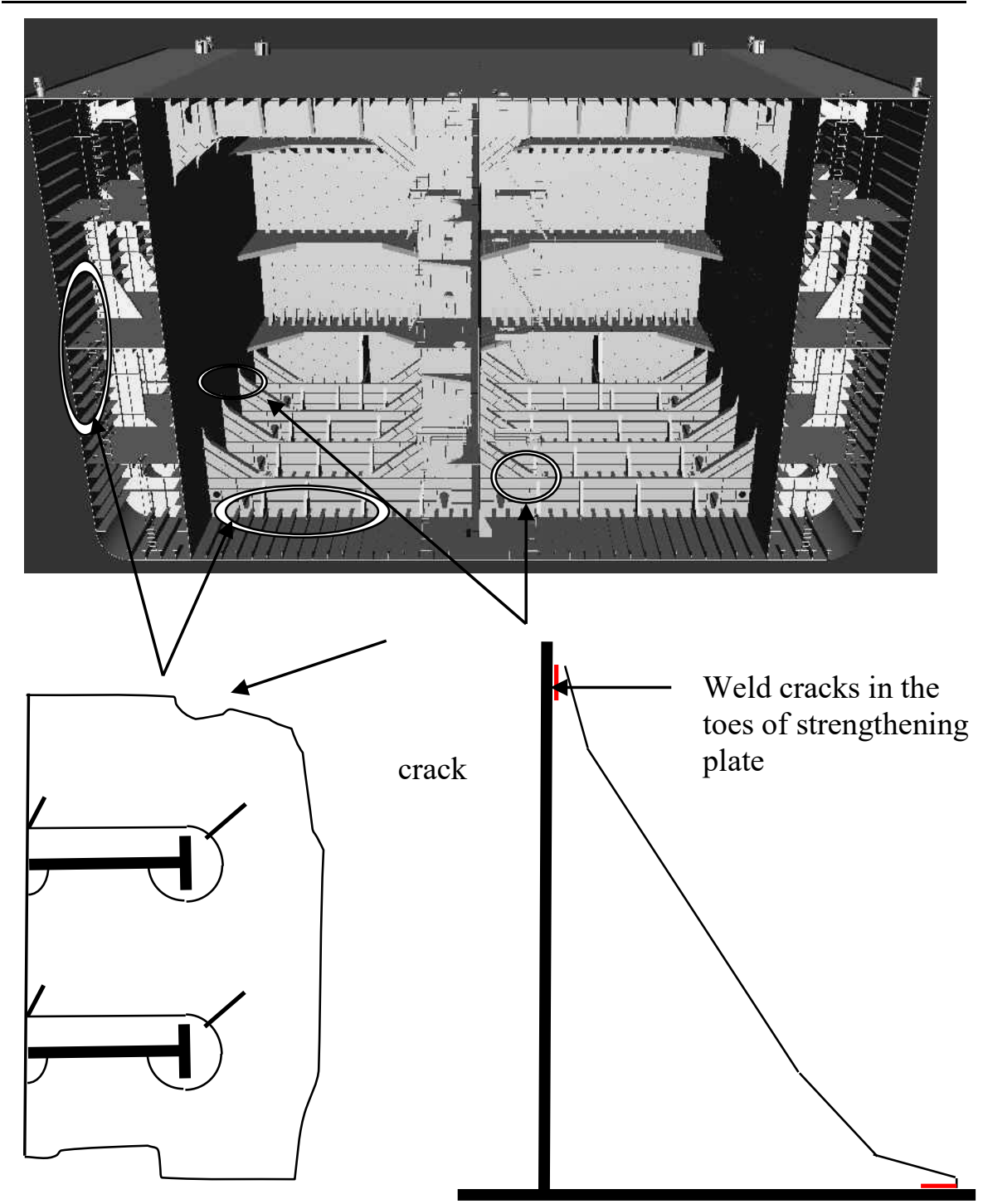

Fig. 1. Cross-section of ship showing two FPSO tanks in the middle and two ballast tanks on the sides.

inspected in the first five years costs $£ 60-70 \mathrm{k}$. This cost rises to $£ 150-200 \mathrm{k}$ to inspect 3 pairs of cargo tanks and 3-4 pairs of ballast tanks after ten years. These costs can be reduced substantially by sending a robot into the tank without first emptying it thereby saving the cost of cleaning and emptying the tanks. 
Automated NDT of floating production storage oil tanks with a swimming and climbing robot 3

Weld cracks are caused by fatigue and are of two types. Low-Cycle fatigue is driven by panel deflection when filling and emptying tanks causes cracks at the toe of a bracket, generally in the secondary material. The drawing on the bottom right in figure 1 shows a bracket with cracks at its toe ends and the location of the brackets in the FPSO tank. High-Cycle fatigue is driven by wave pressure on the side and bottom shell of the tanks. It causes cracks at cut-outs where shell longitudinal strengthening plates connect to cut outs in the frames. Figure 1 is a drawing (bottom left) of cracks at cut outs and the location of longitudinal strengthening plates.

It is also required to test for corrosion caused by coating breakdown on the tank bottom. Pits can develop at the rate of 2-3 mm/year and even faster at the rate of $5 \mathrm{~mm} /$ year if more corrosive crude is present. The bottom plate is usually $18-25 \mathrm{~mm}$ thick.

\section{The inspection environment}

Obtaining access to welds on strengthening plates on the walls and the floors of the tank is not easy. The environment is very cluttered so that a very large walking and climbing robot would be required to step over plates. However, the robot is required to be compact, mass approximately $20 \mathrm{~kg}$, so that it can be inserted through a manhole of minimum diameter $600 \mathrm{~mm}$. In FPSO's owned by BP, the manholes are two elliptical hatches into each cargo tank approx. 900x600mm in size. FPSO's operated by Petrobras have approx. $600 \mathrm{x} 800 \mathrm{~mm}$ openings. The robot should be transportable by one or at most two operators, and should be able to operate between two adjacent longitudinal strengthening plates separated by a distance of $900 \mathrm{~mm}$ with the transverse frames separated by a distance of $4.5 \mathrm{~m}$. Both the walls and the floor are cluttered with strengthening plates so that unhindered robot motion on the walls or the floor by a small robot is not possible.

Access to welds can be obtained by swimming over the plates from one section of the tank to another and then landing on a wall or floor between the plates. The NDT inspection requirement is to inspect vertical welds as well as horizontal welds.

\section{Access to welds with a swimming and climbing robot}

FPSO Tanks in the North Sea are cleaned first with pressurised crude oil to agitate wax and sludge, and then cleaned with hot sea water. The water is 
removed prior to inspection by human operators, normally only a few centimetres are left on the bottom. The water in the tank tends to be fairly clean, though when disturbed can mix with the oil residues. Therefore, an opportunity exists to change the inspection method by filling the tank with water and then gaining access to welds by swimming to a test site. The robot can adhere to the wall and climb to a suitable location to scan a weld with NDT probes deployed by an arm.

Brazilian off-shore FPSO's are not cleaned with hot water because they are operated in higher temperatures. Therefore, they are cleaned with pressurized oil only thus eliminating the cost of establishing a process that cleans the water before returning it to the sea. Here, a swimming robot would have to operate in crude oil and would therefore have to meet intrinsic safety requirements for operation in explosive environments. Provided the cleaning systems are working, there should be very little residue on the floor, though there is always a waxy film. In places there will be a 2-3mm of wax, like shoe polish on the bottom of the tank but not on the side walls.

\section{NDT requirements and proposed techniques}

Work in tanks is always potentially hazardous and thus a system which can minimise the need for personnel entry is obviously beneficial. The development of a remote tool will only improve structural integrity, and thus potentially reduce leakage, if it can deliver a higher level of inspection than is currently achieved.

The deck and bottom plating is normally $20-25 \mathrm{~mm}$ thick. Bottom stiffeners are typically T-shaped with the web $650 \times 15 \mathrm{~mm}$, and the flange $250 \times 25 \mathrm{~mm}$. Side shell stiffeners are generally bulb bars, 200-400wide and $12-15 \mathrm{~mm}$ thick. Apart from butt joints joining plates together, all connections are fillet weld $6-10 \mathrm{~mm}$ throat thickness depending on the section. Welding size is variable and depends on the design. Coating is provided by a paint layer 300-500 microns thick. The NDT is required to identify through thickness cracks that are $5-10 \mathrm{~mm}$ long (these are currently generally found by visual inspection). If smaller cracks can be detected that would be bonus. It is also important to identify coating defects or pitting on the bottom plates.

The project aims to develop an NDT Sensor Payload that is suitable for robotic deployment and that obtains better NDT data in a hazardous environment than possible with manual inspection. Towards this end, array probes are being developed to use the following NDT techniques: Ultra- 
Automated NDT of floating production storage oil tanks with a swimming and climbing robot 5

sonic phased array technique, Eddy current technique, and the ACFM (Alternating Current Field Measurement) technique.

Phased Arrays: Phased array systems offer the possibility of performing inspections with ultrasonic beams of various angles and focal lengths using a single array of transducers [2]. Software control over beam angle and focusing is achieved by application of precisely controlled delays to both the emission pulse and received signal for each element in an array of transducers, hence the term "Phased Array".

Signals from multi-element transducers in the array produce A-Scan responses that are comparable with those obtained using a fixed angle probe with a conventional pulse-echo imaging system. Therefore, imaging and image interpretation remain the same as for a conventional pulse-echo system. The data can be processed to provide top, side and end view images of the inspected volume of material. In addition to standard imaging, a focused beam is created using a few of the many elements contained in a Phased Array probe (up to 128). The beam is then multiplexed to the other elements to allow a high speed scan of the component with no transducer movement along that axis. Flaw detection and sizing ability is only limited by the beam width which, in theory, can be less than $1 \mathrm{~mm}(0.04 ")$, depending on the excitation frequency. Phased-array probes are being designed to be used to scan for defects in fillet welds. They have a stainless steel housing with dimensions of L $60 \mathrm{~mm} \times \mathrm{W} 20 \mathrm{~mm}$ with cable output from the centre on top, with Wedge material being Rexolite $(\mathrm{v}=2350+/-30$ $\mathrm{m} / \mathrm{s}$ ) and the angle is 34 degrees.

The Eddy Current Array technique will be used to inspect the tank floor for corrosion pitting in the presence of sludge and wax. The robot will carry a set of array probes and its motion will result in a surface scan. A feasibility study with a conventional eddy current system shows that the conventional eddy current may work to pick up corrosion type of defect, if all the parameters are optimised. Therefore a system is being developed that consists of a conventional eddy current probe array.

ACFM (Alternating Current Field Measurement) techniques: ACFM is an electromagnetic inspection technique that provides one pass inspection. It has a high tolerance to lift off and requires no electrical contact so that it can be used to detect through coatings. It provides crack detection and sizing and is suitable for weld inspection. To cover the weld cap, toes and HAZ of a welded joint, it is necessary to either use a simple single sensor probe and scan several times or use a multisensor array probe and cover the required area in a single pass. Work is progressing to develop an underwater ACFM array probe that can be deployed on the robotic vehicle. The probe is to be used to detect and size surface breaking cracks at or in 
close proximity to the welds to be inspected. Modifications will be needed to make existing instrumentation intrinsically-safe. The objective is to scan a fillet weld in one pass, accommodate different cap widths, and minimise weight. The current probe design is estimated to weigh approximately $1.2 \mathrm{~kg}$ in air. The maximum dimensions are $87 \mathrm{~mm}$ wide $\mathrm{x} 117 \mathrm{~mm}$ long $\mathrm{x}$ $117 \mathrm{~mm}$ high. The materials have been chosen to be mainly stainless steel for structural parts and PEEK for parts that will not be subject to great loads.

\section{Robot Design}

The robot design selected for construction is shown in figure 2 with the robot travelling on the floor. In swimming mode the bottom side is the one fitted with the scanning arm while the wheels point in the direction of forward motion. This design is a further development of a wall climbing robot called RobTank that has been developed earlier for in-service inspection of oil and chemical storage tanks [3]. The wall-climbing ability of this robot has been tested and demonstrated in water tanks. It can make transitions from a floor to a wall and vice-versa. Further development of this design

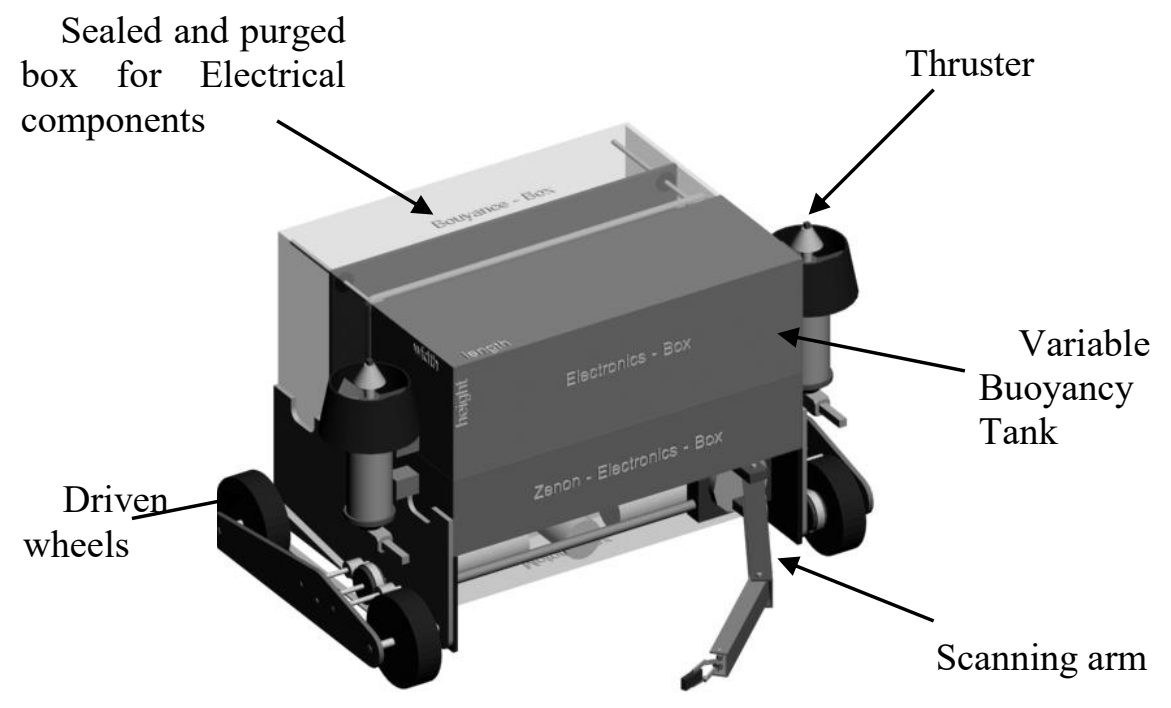

Fig. 2. Conceptual design of FPSO swimming and wall climbing robot 
Automated NDT of floating production storage oil tanks with a swimming and climbing robot 7

has added a variable buoyancy tank that can quickly and accurately control buoyancy around the neutral buoyancy of the robot.

All motors, motion controllers, navigation electronics, NDT flaw detectors are housed in an air pressurized central chamber to prevent the ingress of water through any leaks at the rotating shafts emerging from the central chamber and through NDT sensor probe cables. The reason for placing most hardware systems onboard the robot is to reduce the size of the umbilical cord so that cable management becomes easier.

The outer dimensions of the robot are $(\mathrm{mm}): 540 \mathrm{~L}$ x $300 \mathrm{~W} \times 300 \mathrm{H}$. Its mass in air is $12 \mathrm{~kg}$ and it can carry a payload of $8 \mathrm{~kg}$. On-board embedded servo controllers with encoder feedback control the speed and position of the robot. High level control is from an operators console via RS 485 twin pair communications with on-board controllers.

Both depth and horizontal motion is controlled simultaneously to swim the robot to a test site on a wall. After contact with a wall, thrust forces obtain adhesion to the wall while actuated wheels move the robot on the wall. The robot manoeuvres freely on the wall and can be driven down from a wall to the floor of the tank and back on to it.

After insertion of the robot through a manhole in the top deck, positive or negative buoyancy control is used to swim the robot vertically to a specified depth and to maintain that depth with neutral buoyancy. Two independent, speed controlled thrusters move the robot in a horizontal plane in the forward and reverse direction or rotate it to face in any direction.

Figure 3 shows the prototype swimming and wall climbing robot. Figure 4 shows the robot climbing on the glass wall of a water tank. Adhesion to the wall is obtained via forces generated by two thrusters. The robot is able to change surfaces (floor to wall and back) and climb on the wall without the aid of neutral buoyancy. A variable buoyancy tank is currently being developed to change buoyancy around neutral by obtaining volume change. The tank should enable the robot to swim to a given depth and to be parked on the floor with negative buoyancy when inspecting the floor.

Future work: A scanning arm will be mounted on the robot to deploy ultrasonic phased array probes to perform tests on the toe of fillet welds. Vision systems and range finders will be used to detect tank walls in low light levels and poor visibility. These sensors will be made an integral part of the control system to provide feedback to maintain straight-line motion when moving towards a wall and to avoid collisions. 


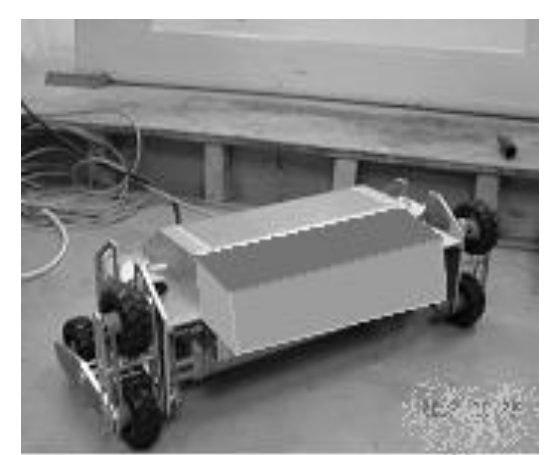

Fig.3. Prototype swimming and wall-climbing robot

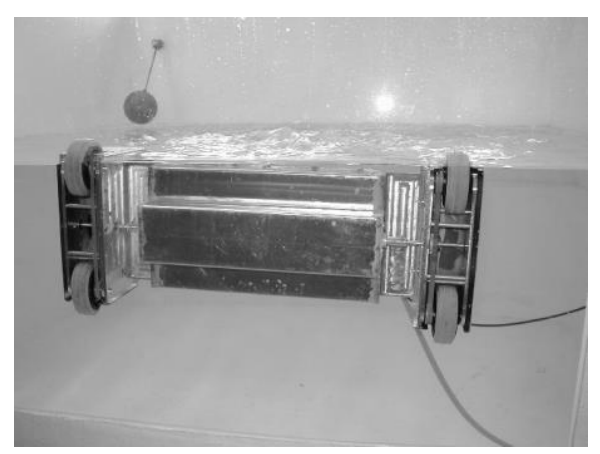

Fig.4. Wall-climbing robot climbing on wall without buoyancy tank

\section{Aknowledgements}

This work is funded by the European Community through the CRAFT project FPSO INSPECT (COOP-2004-508599) [4]with the following partnership: NDT Consultant (UK), TSC Inspection Systems (UK), Isotest Engineering (Italy), Tecnitest Ingenieros (Spain), Spree Engineering Ltd (UK), Miltech Hellas S.A. (Greece), ZENON (Greece), Kingston Computer Consultancy (UK), BP (UK), Petrobras (Brazil) and LondonSouth Bank University (UK). The Project is coordinated and managed by TWI (UK)

\section{References}

1. Shimamura Y. (2002) FPSO/FSO: State of the art, J. Mat. Sci. Technol. 2002, pp 60-70

2. American Society for Nondestructive Testing Handbook (2002), Vol. 7 Ultrasonic Testing.

3. Sattar T.P., Zhao Z., Feng J., Bridge B., Mondal S., Chen S., (2002) Internal In-service Inspection of the floor and walls of Oil, Petroleum and Chemical Storage Tanks with a Mobile Robot, Proc. Of 5th International Conference on Climbing and Walking Robots and the Support Technologies for Mobile Machines, Edited by Philipe Bidaud and Faiz Ben Amar, ISBN 186058380 6, 2002, pp 947-954, Professional Engineering Publishing Ltd. UK.

4. Non-Intrusive In-service Inspection Robotic System for Condition Monitoring of Welds inside Floating Production Storage and Offloading (FPSO) Vessels (2004), EU $6^{\text {th }}$ Framework Programme, Co-operative Research Project, COOP-CT-2004-508599, December 2004. 